[3] Tong B, Liu X, Xiao J, Su G. Immunopathogenesis of Behcet's Disease. Front. Immunol 2019;10:665.

Disclosure of Interests: None declared

DOI: 10.1136/annrheumdis-2020-eular.6572

\section{AB1027 EFFECTIVENESS OF SUBCUTANEOUS ANTI- INTERLEUCINE 6 TREATMENT IN PATIENTS WITH ACTIVE MODERATE-SEVERE GRAVES' ORBITOPATHY REFRACTORY TO CONVENTIONAL THERAPY.}

L. Fernández de la Fuente Bursón ${ }^{1}$, F. J. Toyos Sáenz de Miera' ${ }^{1}$, D. RuizMontesinos $^{1}$, M. Gessa Sorroche ${ }^{2}$, M. C. Díaz Ruiz² ${ }^{2}$ M. J. Madrigal

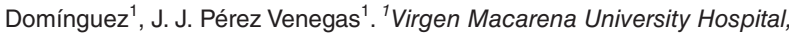
Rheumatology, Seville, Spain; ${ }^{2}$ Virgen Macarena University Hospital, Ophthalmology, Seville, Spain

Background: Graves' Orbitopathy (OG) is the main extrathyroid manifestation of Graves' disease (GD). Up to $25 \%$ of patients have moderate-severe inflammatory activity with a risk of major sequelae. Intravenous (i.v.) glucocorticoids (GC) pulses are the standard therapy. In refractory cases, several classic and some biological immunosuppressantsare used, obtaining variable responses. Interleukin 6 (IL6) is involved in the pathogenesis of OG, which has justified the off-label treatment with Tocilizumab (TCZ) ${ }^{1}$, whose use by route i.v. has shown favorable results in published small case series and a single multicenter trial ${ }^{2}$

Objectives: To analyse the ocular effectiveness of anti-IL6 therapy used subcutaneously (s.c.) in patients with moderate-severe active refractory $O G$ in usual clinical practice.

Methods: Retrospective descriptive observational study of a series of cases of moderate-severe OG patients treated with anti-IL6 s.c. The patient medical records of those who had received at least 1 cycle of anti-IL6 treatment were reviewed (December 2013-December 2019). The primary effectiveness outcome was the change of the Clinical Activity Score (CAS). Favorable response was considered: reduction of $\mathrm{CAS} \geq 2$ points together with obtaining inactivity (CAS $<3)$. Demographic data, personal medical history, clinical aspects of GD, previous therapies and data on the use and safety of anti-IL6 were collected. The SPSS11 package was used for statistical analysis, using non-parametric tests for quantitative variables. The study was approved by the local Ethical Committee.

Results: 12 of the 15 patients ( $80 \%$ ) were women with a mean of 50.27 years (21-72). 60\% $(n=9)$ had smoking history, $40 \%(n=6)$ active. $26.7 \%(n=4)$ were diabetic, all without retinopathy. $100 \%$ of patients received imidazole antithyroid treatment. $46.7 \%(n=7)$ required $\beta$-blockers and $20 \%(n=3)$ diuretics. $66.7 \%$ thyroidectomy $(n=10)$ and $20 \%(n=3)$ decompressive eye surgery and/or blepharoplasty were performed. Thyroid and ocular radiotherapy were used in 2 patients. 3 patients received botox. $80 \%(n=12)$ of them had previously received GC. 93.3\% ( $n=14)$ were naïve to biological therapy, only 1 patient previously used Rituximab. All except one patient who was treated with SRL received TCZ as IL6 therapy. A significant favorable response was obtained in $100 \%$ of the patients $(p=0.008)$, decreasing CAS average from $4.9(2-7)$ to $1.7(0-2)$ at the end of the therapy [Figure 1]. The severity of the OG changed from being moderate in $72.7 \%$ of the patients to mild in $66.7 \%$ of the total. The median time to inactivity was 8 months (2-15). $73.3 \%(n=11)$ of the patients finished the treatment reaching inactive OG, the rest (although inactive) maintained therapy. After 6 months, $100 \%$ of those who completed the treatment remained inactive with average CAS of 1.3 (0-2). Smoking did not influence the response, nor any other variable collected. Adverse events appeared in $26.7 \%(n=4)$ of the cases, all of them mild and without widrawal.

Conclusion: Treatment with anti-IL6 s.c. steadily decreases the clinical activity measured by CAS in patients with moderate-severe refractory OG, despite poor prognosis factors (such as smoking), with a good safety profile.

References:

[1] Taylor PN, Zhang L, Lee RWJ, Muller I, Ezra DG, Dayan CM, et al. New insights into the pathogenesis and nonsurgical management of Graves' orbitopathy. Nat Rev Endocrinol. 2020 Feb;16(2):104-116.

[2] Perez-Moreiras JV, Gomez-Reino JJ, Maneiro JR, Perez-Pampin E, Romo Lopez A, Rodríguez Álvarez FM, et al. Efficacy of Tocilizumab in Patients With Moderate-to-Severe Corticosteroid-Resistant Graves' Orbitopathy: A Randomized Clinical Trial. Am J Ophthalmol. 2018;195:181-90.

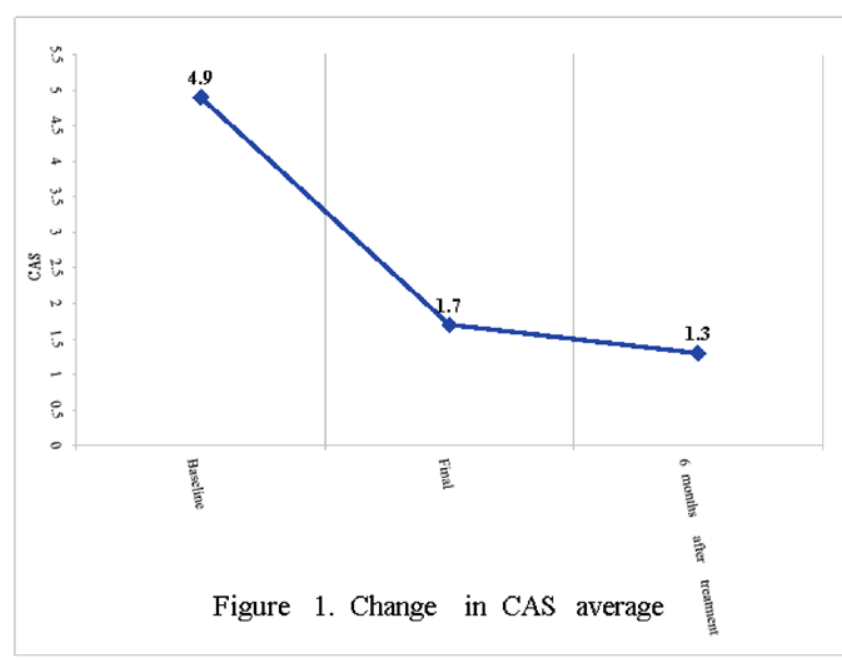

Disclosure of Interests: None declared

DOI: 10.1136/annrheumdis-2020-eular.6201

\section{AB1028 \\ A SINGLE-CENTER EXPERIENCE WITH TRANSIENT OSTEOPOROSIS - PATIENT CHARACTERISTIC AND APPROACH TO THERAPY.}

S. Giryes1, K. Dolnikov ${ }^{1,2}$, A. Balbir-Gurman ${ }^{1,2}$, D. Militianu ${ }^{1,2}$, N. Puchkov1, Y. Braun-Moscovici ${ }^{1,2}$. 1 Rambam Health Care Campus, Haifa, Israel; ${ }^{2}$ The Ruth and Bruce Rappaport Faculty of Medicine, Haifa, Israel

Background: Transient osteoporosis (TOP) or transient bone marrow edema syndrome is an enigmatic condition of unknown etiology first described in pregnant women. Though usually self limited, TOP causes pain and debilitation and predisposes the patient to avascular necrosis or fractures. The course can be protracted and patient may suffer relapses. Diagnostic method of choice is magnetic resonance imaging (MRI). Based on small case series and expert opinion several therapeutic approaches have been proposed to hasten the recovery, including bisphosphonates, calcitonin, teriparatide. However, the literature is scarce and additional experience is needed to promote the understanding of this condition

Objectives: To present our experience with TOP, including patient's characteristic, approach to diagnosis, prognosis and therapy

Methods: It is retrospective, single center study, conducted in Rambam healthcare campus, Haifa, Israel. All the medical files of patients referred to Rheumatology unit between years 2010-2020 were screened for diagnosis of TOP. Search words included: "osteoporosis," "bone marrow edema", "transient osteoporosis". The files were reviewed for patient's characteristics, modality of diagnosis, duration until full recovery, treatments and relapses.

Results: Eight patients with at least one episode of TOP were identified using the search words. Six patients $(75 \%)$ were female. Three female patients developed TOP during or shortly after pregnancy. Two patients - one male and one non pregnant female suffered from TOP after bariatric surgery. One pregnant woman had a strong family history of TOP. The most frequent involved site in order of frequency were: hip (4/8), ankle (3/8) and knee (2/8). Six patients presented with more than one simultaneous site of TOP (hips, knees and ankles). Blood count, liver and Kidney function tests, markers of bone resorption, rheumatoid factor, Anti cyclic citrullinated peptide, Antinuclear antibodies were negative in all of the patients. C-reactive protein was elevated in $4 / 8$ patients, Erythrocyte sedimentation rate was elevated in 2/8 patients. All patients had vitamin $D$ deficiency. The diagnosis was confirmed by MRI. All the patients were treated with vitamin $\mathrm{D}$ and intra-venous Pamidronate, one patient with addition of calcitonin and one patient with addition of intra venous lloprost. Time to recovery ranged from 1.2 to 6 months. The time to recovery was the same in pregnancy related TOP. Recovery was confirmed with follow-up MRI in all the patients. Relapses occurred in $4 / 8$ patients and only one them had pregnancy related TOP. All the patients were treated by multidisciplinary team, including orthopedic surgeon, physiotherapist and psychologist when needed.

Conclusion: Our experience with TOP was enriched in patients presenting with more than one site of disease probably representing referral bias. Pregnancy related TOP was associated with lower risk of relapse. In terms of time to recovery there was no trend between pregnancy related and non related TOP or one site versus several sited TOP. None of the patients developed fracture, advocating in favor of adding bisphosphonates to therapy. Multidisciplinary approach is an essential part of TOP treatment strategy. 